\title{
Canada's oral health professionals and antimicrobial stewardship
}

\author{
Office of the Chief Dental Officer of Canada ${ }^{1}$
}

\begin{abstract}
Antimicrobial resistance (AMR) is a global concern as it poses a serious threat to our capacity to treat common infectious diseases. Canada has been engaged in actions to address the AMR challenge since 1997, and these actions include a four-pillar national strategy: surveillance; stewardship; infection prevention and control; and research and innovation. Dentists play a significant role in contributing to the efforts around these four-pillars, especially that of stewardship. Studies show that antibiotic prescriptions for oral health reasons, are increasing over time, and $60 \%$ to $80 \%$ of antibiotics prescribed in a dental setting are not necessarily clinically indicated. The development, promotion and implementation of initiatives to promote optimal use of antimicrobials across Canada will require collaboration among many stakeholders, including the oral health community. Antimicrobial resistance and antimicrobial stewardship are already being discussed within the dental profession in Canada; however, there is still more work to be done in a variety of areas including, but not limited to, dentist's access to and use of current evidence-based guidelines and prescribing protocols enforced by their governing bodies to ensure appropriate prescribing of antibiotics when necessary, and timely and affordable access to oral health care services by Canadians.
\end{abstract}

This work is licensed under a Creative Commons Attribution 4.0 International License.

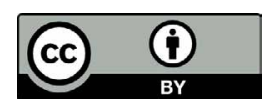

Affiliation

1 Office of the Chief Dental Officer of Canada, Public Health Agency of Canada, Ottawa, ON

Correspondence:

claudia.gorenko@canada.ca

Suggested citation: Office of the Chief Dental Officer of Canada. Canada's oral health professionals and antimicrobial stewardship. Can Commun Dis Rep 2020;46(11/12):376-9.

https://doi.org/10.14745/ccdr.v46i1112a02

Keywords: AMR, stewardship, antibiotics, prescription, best practices, dentists

\section{Introduction}

The discovery and development of antibiotics is considered one of the greatest medical achievements of the $20^{\text {th }}$ century; however, with increased use, bacteria can develop a resistance to antibiotics over time. The World Health Organization (WHO) has made antimicrobial resistance (AMR) a priority. WHO calls for a global coordinated action to minimize the emergence and spread of AMR, and for each country to have a national action plan in place (1). Canada has been engaged in actions to address the challenges of AMR since 1997, and these actions include a four-pillar national strategy: surveillance; stewardship; infection prevention and control; and research and innovation (2-4). As highlighted in the 2019 Chief Public Health Officer of Canada Spotlight Report, Canada has made progress and rates of antibiotic resistance are lower than in many other countries in the world (5). However, there is still work to be done.

As healthcare providers and prescribers, dentists have a significant role to play in contributing to the efforts around the four-pillar national strategy, especially stewardship. Antimicrobial stewardship (AMS) refers to coordinated interventions designed to promote, improve, monitor and evaluate judicious appropriate antimicrobial use to preserve their future effectiveness and to promote and protect human and animal health (2). The following sections will provide a closer look at the prescribing habits in Canadian oral health practice versus that of medical health practitioners and AMS initiatives already underway, and will provide an overview of the AMS work that lies ahead in Canadian dentistry.

\section{Antimicrobial use and prescribing practices}

In Canada, approximately $92 \%$ of antibiotics are used outside of the acute care hospital setting; $89 \%$ are prescribed by physicians, $8 \%$ by dentists and $3 \%$ by nurses, pharmacists and optometrists (2). In dentistry, there are two uses for antibiotics: prophylactic and therapeutic. Prophylactic antibiotics are used to prevent infection and these types of prescriptions are more prevalent than those for therapeutic antibiotics, which are used to treat an existing infection (5). The Canadian Dental Association supports the American Heart Association's guidelines for antibiotic prophylaxis prior to certain oral health procedures to prevent infective endocarditis on high-risk patients only. These guidelines 
state that, due to a growing body of evidence, the risks of taking preventive antibiotics outweigh the benefits for most patients (6). Despite these guidelines, in a retrospective cohort study conducted in the United States (US) looking at data from 2011-2015, dentists in the US were found to often prescribe primary prophylaxis to healthy patients undergoing invasive oral health procedures even though evidence in support of such use is minimal and inconclusive (7). Specifically, the researchers found that more than $80 \%$ of antibiotics prescribed for infection prophylaxis before dental visits were unnecessary (7).

Studies in the United Kingdom and US have shown that between $60 \%$ and $80 \%$ of antibiotics prescribed in a dental setting are not necessarily clinically indicated (7-9). Unfortunately, at this time, there are limited published data on the antibiotic prescribing practices of dentists in Canada. Canadian data that are available show a downward trend in antimicrobial prescribing rate among physicians (10). An increase in prescriptions among dentists (2010-2012) was followed by a generally stable rate from 2012-2015 (10). A study in British Columbia also showed overall antibiotic use and physician-prescribing declined by $18.2 \%$ between 1996 and 2013 (11). However, there was a reported $62.2 \%$ increase in antibiotic prescribing by dentists in British Columbia over the same time period-at a time when the use of recommendations and guidelines should have resulted in a decline (11).

The Canadian Dental Association (CDA) attempted to document prescribing practices of dentists across Canada in 2017. Results of a web-based survey showed that most dentists in Canada reported prescribing antibiotics according to the best available evidence and clinical guidelines (12). The results also indicated that there were concerns including, but not limited to, overuse of certain types of antibiotics, discrepancies regarding medical conditions and dental procedures requiring antibiotic prophylaxis for the prevention of infective endocarditis and dental conditions requiring therapeutic antibiotics. Another concern was an apparent lack of awareness, among certain dentists, to changes in antibiotic prescribing guidelines (12). While the survey was based on self-reported information and the sample size was small ( $n=1,035$, representing a $16.5 \%$ response rate), the results point to the need for more research to better understand the prescribing practices of Canadian dentists.

Numerous factors, over and above evidence and best-practice, contribute to the decision to prescribe an antibiotic. These factors may include recommendations from other health professionals, patient expectations, unclear, outdated or changing guidelines or lack of awareness of recent guidelines, diagnostic uncertainty and time constraints (5). According to a study done by Suda et al. (7), reasons for higher antibiotic prescribing rates among dentists included increasing use of dental implants, an aging population, underinsurance driving antibiotics as an oral surgery substitute, slow adoption of new guidelines, lack of awareness of the role of dentists in antimicrobial resistance, and physician and patient pressure.
These characteristics are similar to those associated with physician antibiotic overprescribing (7). In addition, a scoping review found that reasons for prescribing therapeutic antibiotics included limited time for emergency appointments, time constraints, and uninsured patients who were unable to afford appropriate treatment (13). Nonetheless, similar to other prescribing healthcare professionals, the challenge for dentists lies in ensuring that they are prescribing antibiotics only when necessary and in strict compliance with the recommended dosage and duration for that antibiotic (aiming for the shortest possible time for the required therapeutic effect).

Contributing to the issues around AMR, some patients see physicians at hospital emergency departments (EDs). Between 2001 and 2010, visits to US EDs by 20-29 year olds accounted for $42 \%$ of all ED toothache visits, which ranked as the fifth most common reason for any ED visit and third most common for uninsured ED visits in this age group $(14,15)$. In 2019, another group of researchers conducted a scoping review using Canadian and US data to map out preliminary factors associated with patients' use of EDs for non-traumatic dental problems (15). While the researchers stated several limitations, their preliminary results showed that patients visit EDs due to demographics, accessibility, economic and social influences with income and inability to afford care as the most common factors.

\section{Dental stewardship initiatives on antimicrobial resistance in Canada}

Some stewardship initiatives are already underway to raise awareness and educate dental professionals on AMR. For example, AMR and AMS are already being discussed within the dental profession in Canada. The CDA is raising awareness of AMR, encouraging the mobilization of Canadian oral health professionals and publishing articles on AMR in the CDA magazine (16). The CDA has also been a participant in the Public Health Agency of Canada (PHAC)-sponsored national AMR collaborations. Within PHAC, the Office of the Chief Dental Officer of Canada is also working with the PHAC AMR team and with key national oral health stakeholders to enable the profession to align with best prescribing practices in order to mitigate the risks of AMR. The Canadian Association of Hospital Dentists will be participating in the HealthCareCAN Action Roundtable to develop a National Antimicrobial Stewardship Action Plan. It is also working with the dentists and infectious disease physicians to communicate the critical importance of responsible antimicrobial use (17).

In terms of resources for clinicians, in the US, the Massachusetts Department of Public Health has developed an Antibiotic Stewardship Toolkit for Oral Health Clinicians, which consists of two short YouTube ${ }^{\circledR}$ videos in addition to webinars (18). In Canada, PHAC has contributed to the sponsorship of training modules on AMR, giving Canadian dentists access to focused continuing education through the University of Waterloo Online 
training modules (19). Dentists can also guide their patients toward easy-to-read information about AMR within websites from "Do Bugs Need Drugs" (20) and "Choose Wisely Canada/ Antibiotics Wise" (21).

Moving forward, different approaches should be considered by the dental profession to address AMR. The development, promotion and implementation of initiatives to promote optimal use of antimicrobials across Canada will require collaboration among many stakeholders (22). Governments should further explore oral health care access disparities and inequalities faced by segments of the population in order to prevent unnecessary visits to see physicians and other medical providers for oral health issues $(14,15)$. This would reduce the unnecessary prescription of antibiotics as outlined above in, for example, EDs (14). Federal, provincial and territorial governments and their respective regulatory bodies also play a key role in the shared responsibility of monitoring and evaluating guidelines, an essential component of AMS strategies (22). WHO and US Centers for Disease Control and Prevention (CDC) are formulating policies on AMS (23). Examples of CDC's core elements of outpatient AMS are accountability, provision of training, monitoring and reporting on prescribing patterns, and education for both clinicians and patients (23). Governments and dental associations and governing bodies can use these as a starting point.

There is support for AMS initiatives at the government and dental association levels $(2,6,22)$. Continuing education opportunities, professional guidelines, and awareness-raising should be used and promoted among dentists in addition to additional research in Canada to better understand antibiotic prescribing habits of dental clinicians.

\section{Competing interests}

None.

\section{Funding}

This work is supported by the Public Health Agency of Canada.

\section{References}

1. World Health Organization. Antimicrobial Resistance. Geneva (Switzerland): WHO; July 2020. https://www.who.int/ news-room/fact-sheets/detail/antimicrobial-resistance

2. Public Health Agency of Canada. Tackling Antimicrobial Resistance and Antimicrobial Use: A Pan-Canadian Framework for Action. Component 3: Stewardship. Ottawa (ON): PHAC; 2017. https://www.canada.ca/en/ health-canada/services/publications/drugs-health-products/ tackling-antimicrobial-resistance-use-pan-canadian-framew ork-action.html\#a3.3
3. Public Health Agency of Canada. Office of Audit and Evaluation. Evaluation of the Coordination of Antimicrobial Resistance (AMR) Activities. Ottawa (ON): OAE/PHAC; 2019. https://www.canada.ca/en/public-health/corporate/ transparency/corporate-management-reporting/ evaluation/report-evaluation-coordination-antimicorbial-res istance-activities.html

4. National Collaborating Centre for Infectious Diseases. Surveillance of Antimicrobial Resistance and Antimicrobial Utilization in Canada. NCCID; 2014. https://nccid.ca/publications/surveillance-of-antimicrobia I-resistance-and-antimicrobial-utilization-in-canada/

5. Public Health Agency of Canada. Handle with Care: Preserving Antibiotics Now and into the Future; Chief Public Health Officer of Canada's 2019 Spotlight Report. Ottawa (ON): PHAC, 2019 (accessed 2020-06-12).

https://www.canada.ca/content/dam/phac-aspc/documents/ corporate/publications/chief-public-health-office

r-reports-state-public-health-canada/preserving-antibiotics/ Final_CPHO_Report_EN_June6_2019.pdf

6. Oberoi SS, Dhingra C, Sharma G, Sardana D. Antibiotics in dental practice: how justified are we. Int Dent J 2015;65(1):4-10. DOI PubMed

7. Canadian Dental Association. CDA Position on Prevention of Infective Endocarditis. CDA; 2014 (accessed 2020-09-02). https://www.cda-adc.ca/en/about/position_statements/ infectiveendocarditis/

8. Suda KJ, Calip GS, Zhou J, Rowan S, Gross AE, Hershow RC, Perez RI, McGregor JC, Evans CT. Assessment of the appropriateness of antibiotic prescriptions for infection prophylaxis before dental procedures, 2011-2015. JAMA Netw Open 2019;2(5):e193909. DOI PubMed

9. Patrick A, Kandiah T. Resistance to change: how much longer will our antibiotics work? Fac Dent J. 2018;9(3):104-11. DOI

10. Thompson W, Tonkin-Crine S, Pavitt SH, McEachan RR, Douglas GV, Aggarwal VR, Sandoe JA. Factors associated with antibiotic prescribing for adults with acute conditions: an umbrella review across primary care and a systematic review focusing on primary dental care. J Antimicrob Chemother 2019;74(8):2139-52. DOI PubMed

11. Public Health Agency of Canada. Canadian Antimicrobial Resistance Surveillance System. Ottawa, 2017 (accessed 2020-09-01). http://www.canada.ca/en/public-health/ services/publications/drugs-health-products/ canadian-antimicrobial-resistance-surveillance-system2017-report-executive-summary.html

12. Marra F, George D, Chong M, Sutherland S, Patrick DM. Antibiotic prescribing by dentists has increased: why? J Am Dent Assoc 2016;147(5):320-7. DOI PubMed

13. Canadian Dental Association. The antibiotic prescribing practices of dentists in Canada. Can Dental Assoc, 2018;5(1):24-7. https://www.cda-adc.ca/en/services/ essentials/2018/issue1/24/ 
14. Stein K, Farmer J, Singhal S, Marra F, Sutherland S, Quiñonez C. The use and misuse of antibiotics in dentistry: A scoping review. J Am Dent Assoc 2018;149(10):869-884.e5. DOl PubMed

15. Lewis CW, McKinney CM, Lee HH, Melbye ML, Rue TC. Visits to US emergency departments by 20- to 29-year-olds with toothache during 2001-2010. J Am Dent Assoc 2015;146(5):295-302.e2. DOI PubMed

16. Canadian Dental Association. CDA essentials. Antimicrobial Resistance: Curbing the Trend. CDA; 2017. https://www. cda-adc.ca/en/services/essentials/2017/issue1/files/assets/ common/downloads/publication.pdf

17. Canadian Association of Hospital Dentists. Antibiotic Stewardship. https://cahd-acdh.ca/antibiotic-stewardship/

18. Kennedy E, Klevens RM. Massachusetts Department of Public Health, Bureau of Infectious Disease and Laboratory Sciences. Antibiotic Stewardship Toolkit for Oral Health Clinicians. MDPHGIS; 2020. http://bit.ly/ DentalStewardshipInformation
19. University of Waterloo. School of Pharmacy. Antimicrobial Stewardship in Primary Care Continuing Education Program (accessed 2020-08-10). https://uwaterloo.ca/ pharmacy/degrees-and-professional-development/ distance-education-continuing-professional-development/ antimicrobial-stewardship-primary-care-continuing-education

20. Alberta Health Services and the British Columbia Centre for Disease Control. Resources for Dentists and Dental Hygienists. AHS and BCCDC; 2019. http:// www. dobugsneeddrugs.org/health-care-professionals/ resources-dentists-dental-hygienists/

21. Antibioticswise.ca. Antibiotics and Dental Care. https://antibioticwise.ca/topics/antibiotics-and-dental-care/

22. Pan-Canadian Public Health Network. Antimicrobial Stewardship. PHNC; 2016. http://www.phn-rsp.ca/pubs/ anstew-gestan/pdf/pub-eng.pdf

23. Teoh L, Thompson W, Suda K. Antimicrobial stewardship in dental practice. J Am Dent Assoc 2020 Aug;151(8):589-95. DOI PubMed

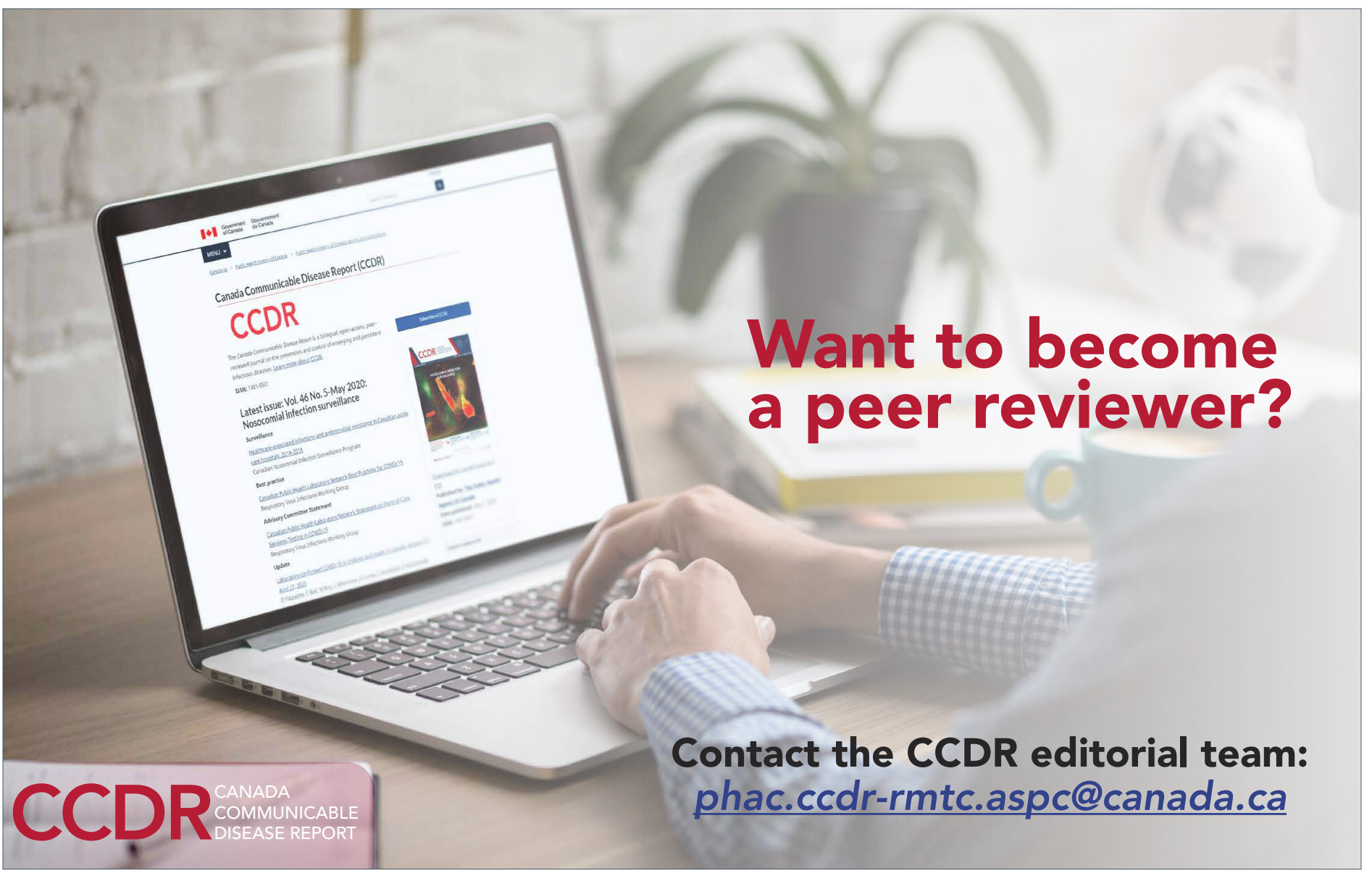

